\title{
Studies on the Conjugating Activity of Bile Acids in Children
}

\author{
SHIN-ICHI NIIJIMA \\ Department of Pediatrics, Juntendo University School of Medicine, Tokyo, Japan
}

\begin{abstract}
The unconjugated and conjugated bile acid levels in sera of 98 normal children and nine normal adults were measured by high performance liquid chromatography. The results showed that the mean total bile acid level was high, $11.0 \pm 8.7 \mu \mathrm{mol} /$ liter (1 SD) during the neonatal period ( $0-4$ wk) and then gradually decreased with age. The ratio of the concentration of conjugated bile acids to total bile acids in serum was as high as $90 \%$ or more in infants under $1 \mathrm{yr}$ of age and slowly decreased with age. The mean ratio of cholic acid to chenodeoxycholic acid was high $(1.7 \pm 1.1)$ during the neonatal period but decreased after 3 months to the adult level $(0.4 \pm 0.2)$. The mean ratio of glycine conjugated bile acids to taurine conjugated bile acids was $3.0 \pm 3.1$ during the neonatal period and the ratio during the 1st month of life was significantly lower than that after that period with little further change at any age. The mean ratio of the concentration of secondary bile acids to primary bile acids showed significantly lower values in infants less than 1 yr of age. The main bile acid was glycocholic acid in the neonatal period but after 1-3 months glycochenodeoxycholic acid predominated. With age, the serum bile acid pattern which was characteristic in infancy gradually approached that of adults. (Pediatr Res 19: 302-307, 1985)
\end{abstract}

\section{Abbreviations}

CA, cholic acid

CA/CDCA, cholid acid/chenodeoxycholic acid

CDCA, chenodeoxycholic acid

DCA, deoxycholic acid

GCA, glycocholic acid

GCDCA, glycochenodeoxycholic acid

GDCA, glycodeoxycholic acid

GLCA, glycolithocholic acid

G/T, glycine conjugated bile acids/taurine conjugated bile acids

GUDCA, glycoursodeoxycholic acid

HPLC, high performance liquid chromatography

LCA, lithocholic acid

$\mathbf{S} / \mathbf{P}$, secondary bile acids/primary bile acids

TCA, taurocholic acid

TCDCA, taurochenodeoxycholic acid

T/D, trihydroxy bile acids/dihydroxy bile acids

TDCA, taurodeoxycholic acid

TLCA, taurolithocholic acid

TUDCA, tauroursodeoxycholic acid

UDCA, ursodeoxycholic acid

Received April 19, 1984; accepted November 1, 1984.

Reprint requests Dr. Shin-ichi Niijima, Department of Pediatrics, Juntendo University, School of Medicine, 3-1-3 Hongo, Bunkyo-ku Tokyo 113, Japan.
Bile acids are synthesized from cholesterol in the liver, conjugated with glycine or taurine before secretion into bile, and circulate in the enterohepatic circulation (10). Major bile acids include the unconjugated, glycine conjugated and taurine conjugated CA, CDCA, DCA, LCA, and UDCA (23). Bile acids leak into the peripheral circulation normally in small amounts (22), which qualitatively and quantitatively change in different hepatobiliary diseases (15).

It has been reported that there is considerable difference in the bile acid metabolism between the fetal and neonatal period and after infancy. In the fetal and neonatal period, $3 \beta$-hydroxy-5cholenoic acid and LCA are synthesized as primary bile acids in addition to CA and CDCA $(3,16)$. The taurine conjugated bile acids are more predominant than glycine conjugated bile acids $(5,7,19)$, and the ratio is later reversed. However, there has been only one report from animals (6) as to whether or not the conjugating activity of bile acids is satisfactory in the neonatal period in which the liver has not developed fully.

For the measurement of bile acids, gas-liquid chromatography has been widely used as a sensitive and reliable method. For routine analysis this method has some shortcomings, e.g. the complex procedure requires a large amount of blood. In recent years, radioimmunoassay has been used for serum bile acids determination $(1,4,9)$ and has provided information on bile acid conjugates. HPLC using a column with immobilized $3 \alpha-$ hydroxysteroid dehydrogenase is suitable for analyzing small amounts of serum for unconjugated and conjugated bile acids separately $(20,21)$ and was, therefore, used to investigate the conjugation pattern of bile acids by age in normal infants and children.

\section{MATERIALS AND METHODS}

Subjects. Ninety-eight infants and children without symptoms or signs of hepatobiliary or gastrointestinal diseases, aged 0-4 wk, 1-3 months, 4-11 months, 1-3 yr, 4-6 yr, 7-9 yr, and 1015 yr were investigated. All infants were mainly bottle-fed. In addition, analyses were performed in nine healthy adults.

Serum samples of $0.5 \mathrm{ml}$ were collected by venipuncture after an overnight fast except for infants younger than 3 months, who were investigated $4 \mathrm{~h}$ after the latest meal.

Extraction of bile acids from serum. The sample was treated as shown in Figure 1. Ethanol ( $2.5 \mathrm{ml})$ was added to $0.5 \mathrm{ml}$ of serum and this mixture was stirred for $1 \mathrm{~min}$ in a water bath $\left(85^{\circ} \mathrm{C}\right)$, followed by centrifugation ( $3500 \mathrm{rpm}$ for $\left.5 \mathrm{~min}\right)$. This procedure was repeated three times, and the combined supernatants were concentrated to dryness under reduced pressure. These extracts were dissolved in $100 \mu \mathrm{l}$ of methanol, and $10 \mu \mathrm{l}$ of this solution was used for analysis with an HPLC system [model BA110C, Japan Spectroscopic Co., Ltd. (Jasco)], including a commercial column for separating bile acids (Jasco Bilepak) and a column packed with immobilized $3 \alpha$-hydroxysteroid dehydro- 


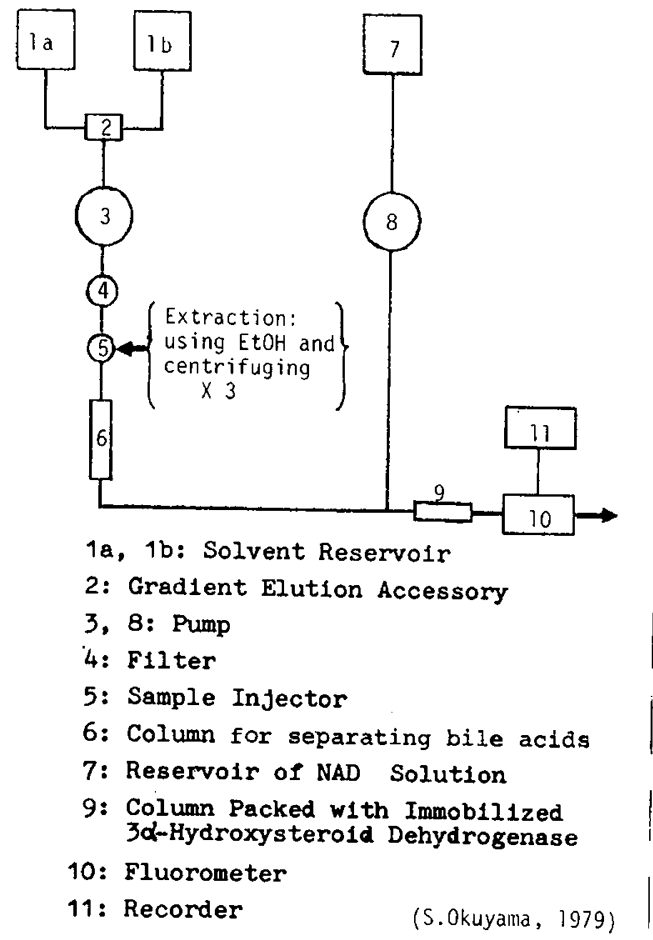

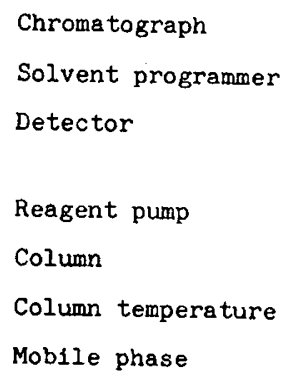

\section{Flow rate}

Immob1lized enzyme column : Jasco Enzymepak-HSD

Column temperature

Reagent

Reagent flow rate

: NAD Solution

$: 0.5 \mathrm{ml} / \mathrm{min}$

Fig. 1. Diagram of the system and chromatographic conditions of HPLC.

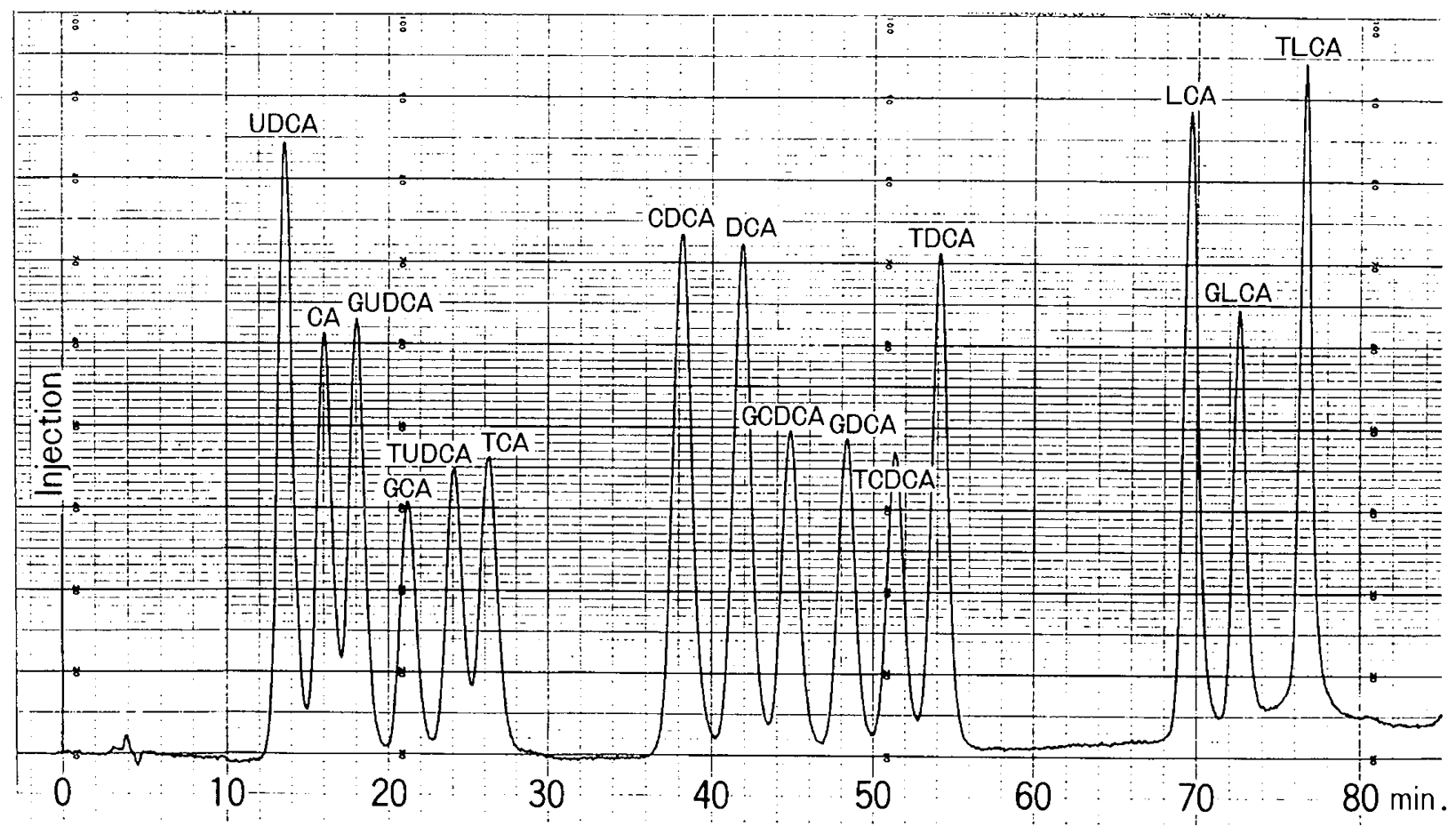

Fig. 2. HPLC analysis of the standard solution of individual bile acids.

genase, (Jasco) with a mobile phase of acetonitrile and phosphate buffer (Fig. 1).

The column preparation of enzyme column was as follows: A suspension of aminopropyl-CPG $(300 \mathrm{mg}$ ) and $3 \mathrm{ml}$ of $2.5 \%$ glutaraldehyde in $\mathrm{H}_{2} \mathrm{O}$ was degassed for 30 min at room temperature, then allowed to stand for $60 \mathrm{~min}$ at $1 \mathrm{~atm}$. The residual CPG glass, washed with distilled water, was added to $10 \mathrm{mM}$ $\mathrm{KH}_{2} \mathrm{PO}_{4}$ solution $(1.5 \mathrm{ml}, \mathrm{pH} 7.80$ ) of $3 \alpha$-hydroxysteroid dehydrogenase $(15 \mathrm{mg})$. The $\mathrm{pH}$ of the solution was adjusted to $\mathrm{pH}$ 7.80 with aqueous $\mathrm{NaOH}$ solution because this enzyme is stable in basic media. This suspension was degassed again for $30 \mathrm{~min}$ in an ice bath and then kept for $30 \mathrm{~min}$ at $1 \mathrm{~atm}$. Chemically bonded CPG was well washed with distilled water and packed in

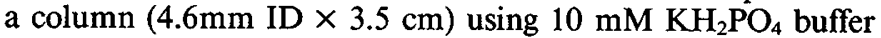
IpH 7.80) containing $1 \mathrm{mM}$ EDTA and $0.05 \%$ 2-mercaptoethanol $(\mathrm{v} / \mathrm{v})$.

Figure 2 shows a chromatogram of standard mixture of bile acids including unconjugated, glycine and taurine conjugated forms of five bile acids (UDCA, CA, CDCA, DCA and LCA), about $50 \mu \mathrm{mol} /$ liter of each. The variability in peak heights 
represents differences in detector responce. The greatest relative deviations for the peak height and the retention time are $3.8 \%$ (for TCA) and $0.53 \%$ (for TCA), respectively. The recovery of each bile acid was more than $88 \%(20,21)$.

Statistical analyses were performed with Student's $t$ test and Mann-Whitney's U test.

\section{RESULTS}

The mean values of total bile acid concentrations, unconjugated bile acids, and percentage conjugated, as well as different ratios, are given in Table 1.
Total bile acid levels. The average total bile acid level was the highest, $11.0 \pm 8.7 \mu \mathrm{mol} /$ liter, during the neonatal period $(0-4$ wk) and gradually decreased with age, becoming $2.8 \pm 1.3 \mu \mathrm{mol} /$ liter in adults. The adult level was significantly lower than values during infancy and children less than $3 \mathrm{yr}$ of age $(p<0.05$ and $<0.01$ for subjects in groups less than $3 \mathrm{yr}$ ). There were no significant differences between children more than 4 yr of age and adults, but levels showed a tendency to decrease with age.

Unconjugated bile acid levels. The mean concentration of unconjugated bile acids was significantly higher, $1.2 \mu \mathrm{mol} / \mathrm{liter}$ in adults compared to newborns $(p<0.05)$. There were no significant differences between the different age groups except

Table 1. The mean ( $\pm S D$ ) concentration of total and free bile acids $(B A)$, the percentage conjugated bile acids and the CA/CDCA, $G / T, S / P$, and $T / D$ ratios in serum of infants and children

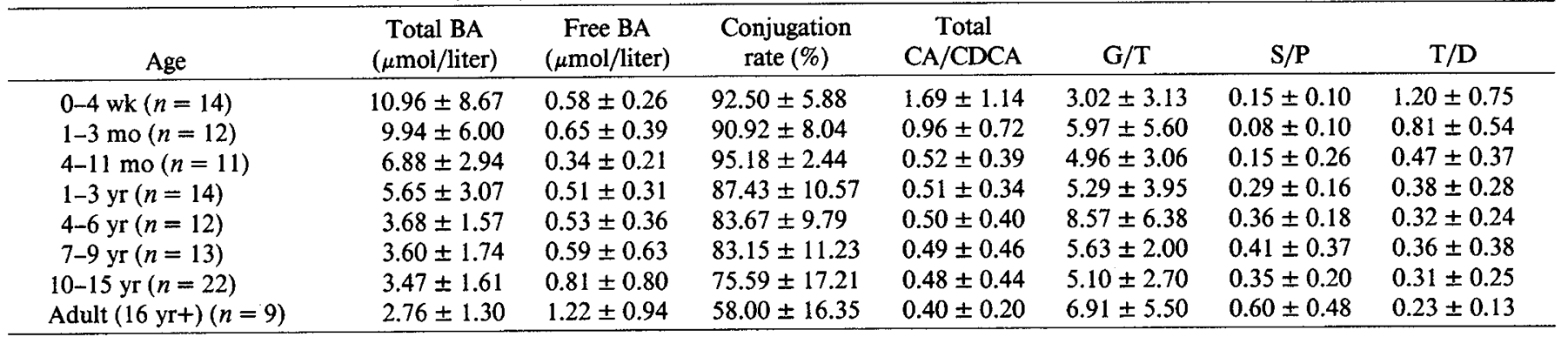

Table 2. The levels of free and conjugated individual bile acids in infants and children (mean $\mu$ mol/liter $\pm S D$ )

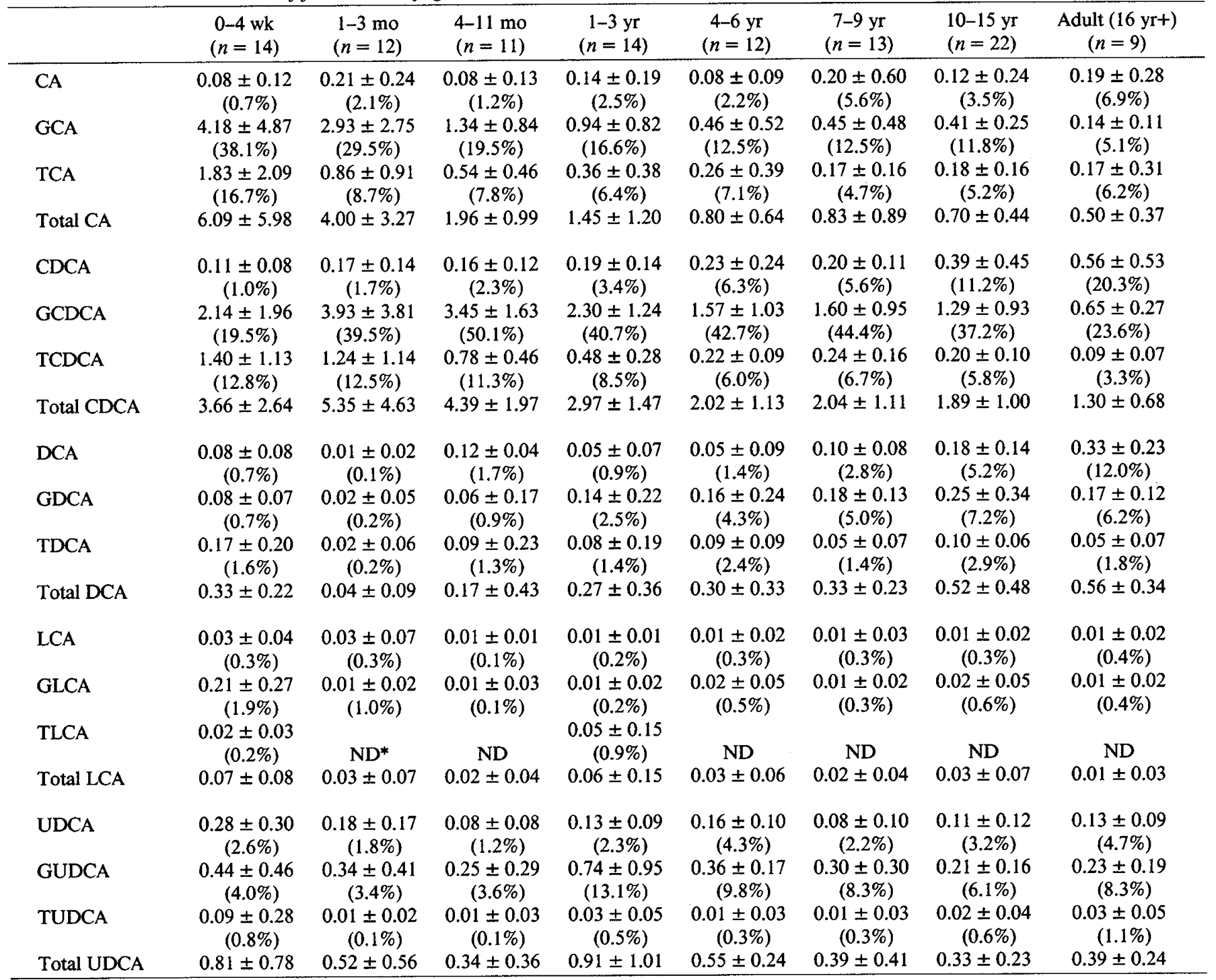

* Not done. 


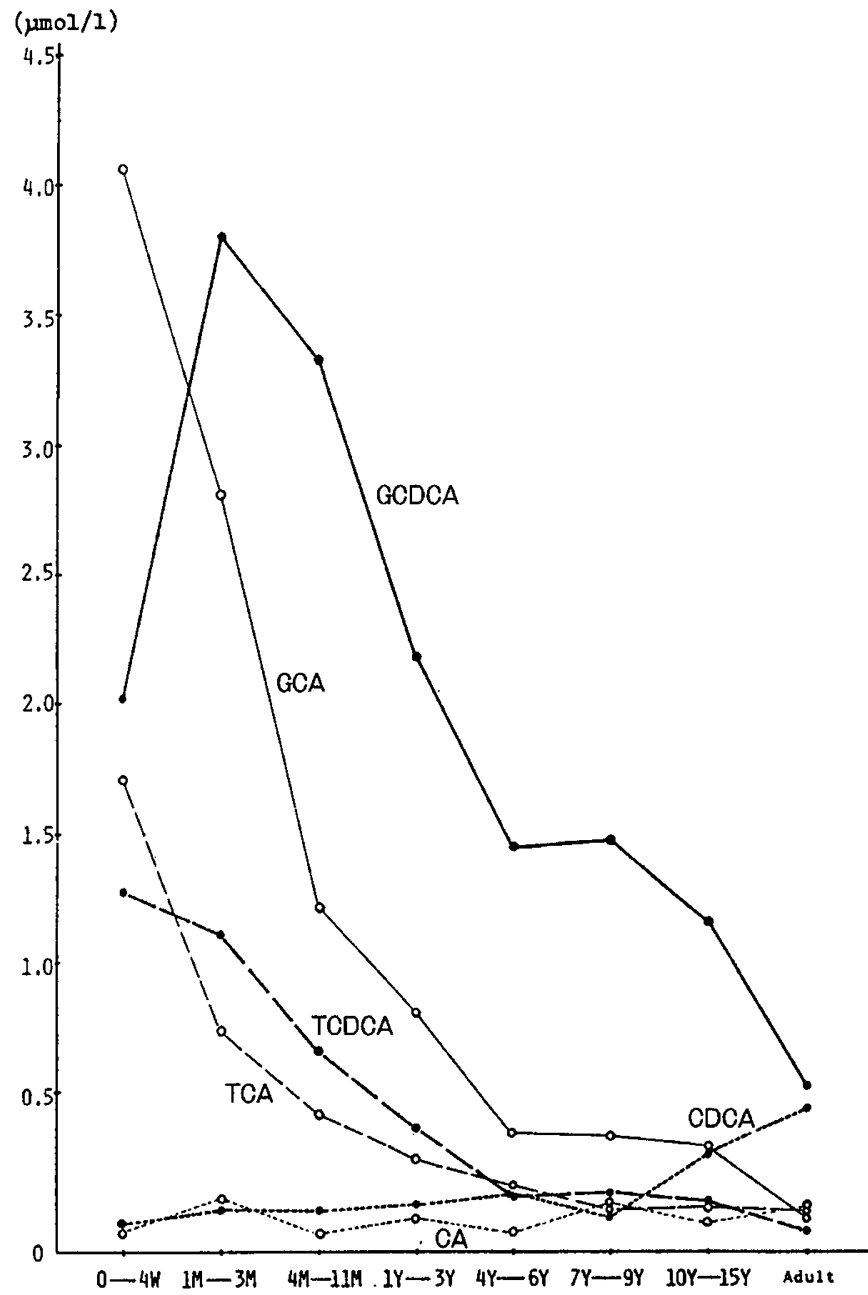

Fig. 3. Primary bile acid levels in sera of normal children at different ages. neonatal period and 4-11 months $(p<0.05)$. The ratio of levels of conjugated bile acids to total bile acids in serum was more than $90 \%$ during the 1 st yr of life. The adult level of $58.0 \pm$ $16.4 \%$ was significantly lower than values during infancy and childhood in all age groups $(p<0.001$ up to $9 \mathrm{yr} ; p<0.0510$ $15 \mathrm{yr}$ ).

$G / T$ ratio. The average ratio of $\mathrm{G} / \mathrm{T}$ showed the lowest value of 3.0 during the neonatal period. Due to the great individual variation in each age group that value was not significantly different from that in older infants or adults (Table 1).

$T / D$ ratio. The average $\mathrm{T} / \mathrm{D}$ ratio showed the highest value of 1.2 during the neonatal period. After 4 months of age there were no significant differences from the value in adults (Table 1). These results were almost parallel to changes in the ratio of $\mathrm{CA} /$ CDCA, which was the highest, 1.7 during the neonatal period ( $p$ $<0.01$ compared to adults or infants more than 4 months of age), and then rapidly decreased to values which after 1 month of age were not significantly different from those in the adults (Table 1).

$S / P$ ratio. The average ratio of $\mathrm{S} / \mathrm{P}$ showed significantly lower values in infants less than $1 \mathrm{yr}$ of age $(p<0.01$ less than 3 months; $p<0.05$ less than 11 months). There was no significant difference between children more than $1 \mathrm{yr}$ of age and adults (Table 1).

Individual bile acid levels. The changes with age in 15 kinds of bile acids and their conjugates are shown in Table 2 and Figure 3. For the comparison chromatograms are shown for a 1month-old boy (Fig. 4) and a 13-yr-old girl (Fig. 5).

The average total CA level showed the highest value of 6.1 $\mu \mathrm{mol} /$ liter during the neonatal period and gradually decreased to the lowest value of $0.5 \mu \mathrm{mol} /$ liter in adults. The adult level was significantly lower than values for infants less than 1 yr of age $(p<0.02)$. The concentration of unconjugated CA was not significantly different among the different age groups. In the neonatal period the main bile acid was GCA, the mean value being $4.2 \mu \mathrm{mol} / \mathrm{liter}$. After $1-3$ months of age GCDCA predominated.

The average total CDCA level showed the lowest value of 1.3 $\mu \mathrm{mol} /$ liter in adults. This level was significantly lower than values

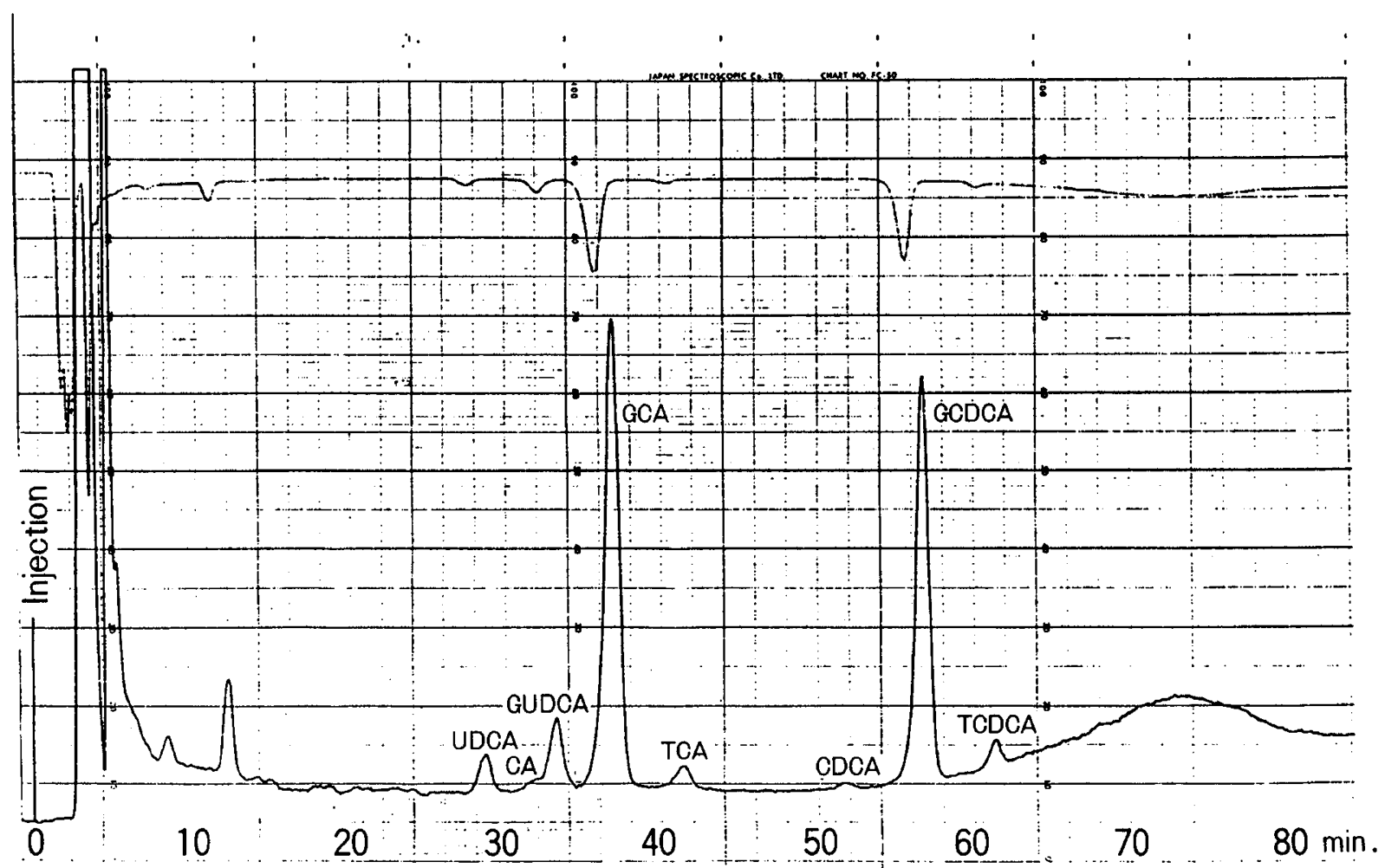

Fig. 4. The chromatogram for serum bile acids in a 1-month-old normal child. 


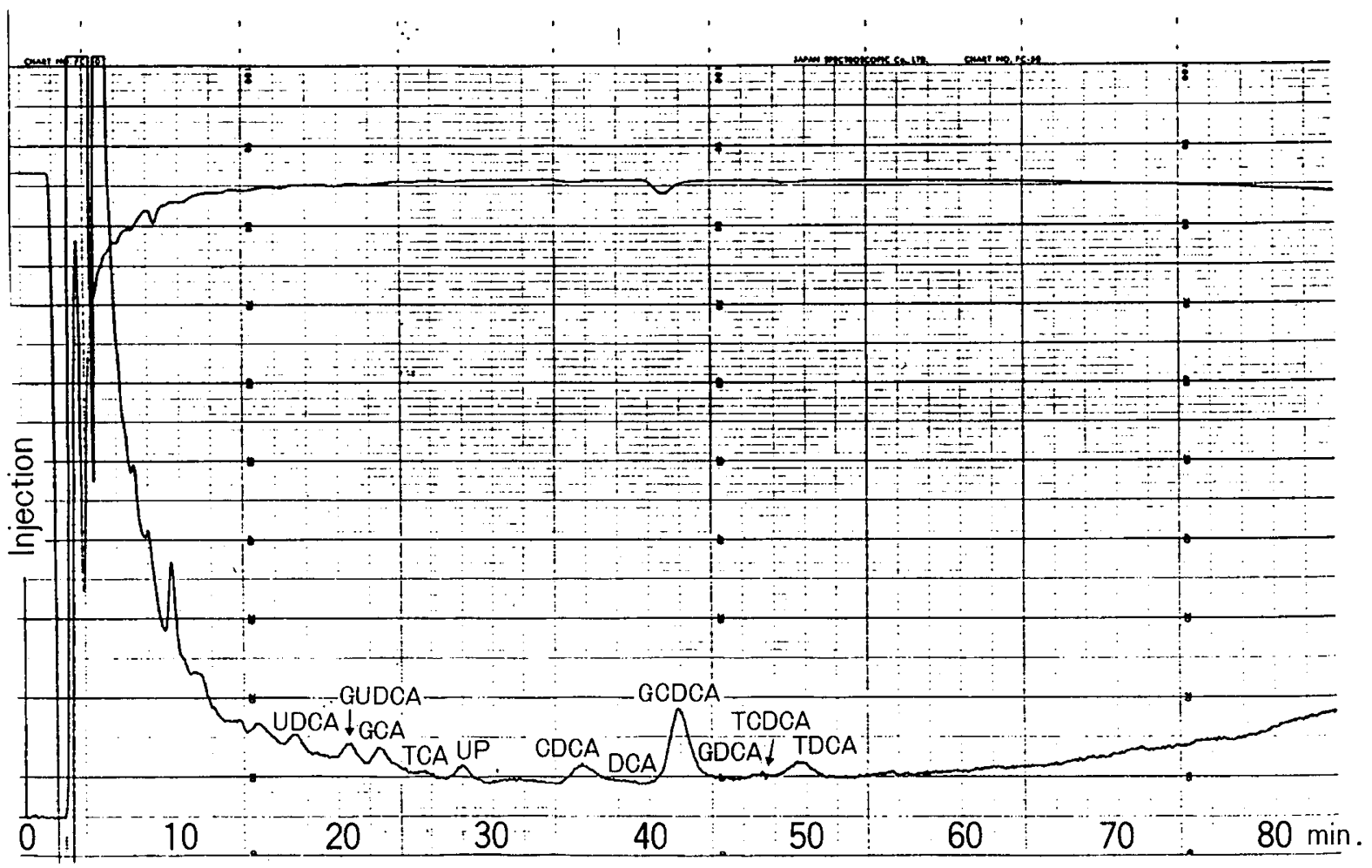

Fig. 5. The chromatogram for serum bile acids in a 13-yr-old normal child.

for infants less than 3 yr of age $(p<0.02)$. There were no significant differences among the different age groups in levels of unconjugated CDCA. Taurine conjugated CDCA decreased significantly with age.

The average total DCA level was not significantly different among the different age groups, but the concentration of unconjugated DCA was significantly higher in the adult than in children less than 9 yr of age $(p<0.01)$. The levels of UDCA and LCA did not change significantly during infancy and childhood.

\section{DISCUSSION}

Arisaka (1) used radioimmunoassay to measure serum GCA levels in infants and children, reporting that the normal value of GCA was higher at a younger age and that the GCA level in the neonatal period was five to six times that in children more than $2 \mathrm{yr}$ of age. It has also been reported by others that CA and CDCA levels in serum were high in the neonatal period compared to those in other age groups $(4,9)$. Our findings confirm these results, which may be due to prematurity of liver function or to the effect of asphyxia at birth during this period of life $(1,4,9$, 24). The conjugation of bile acids in the liver was presumed to be low in the neonatal period when we started this study. By using HPLC, we could establish that the percentage of serum conjugated bile acids was as high as 90 or more in the neonatal period in which the total bile acid levels are high. Subsequently, the percentage of conjugated bile acids showed a tendency to decrease with age. Thus the conjugation of bile acids, as reflected in serum levels, shows a good capacity for conjugating bile acids with the amino acids glycine and taurine in the liver at a time when total bile acids are increased. This is probably one protective factor against the toxicity of bile acids which has been demonstrated in animal experiments $(2,11-14,18)$. The tendency of unconjugated bile acid levels in serum to increase with age is probably due to increasing deconjugation by intestinal bacterial flora (17).

Encranz and Sjövall (7) compared conjugated bile acids in duodenal juice of neonates to adults by thin layer chromatogra- phy and showed that taurine conjugated bile acids predominated in neonates. Nittono et al. (19) determined bile acids in gallbladder contents of premature babies and neonates by HPLC and reported that TCA and TCDCA accounted for more than $70 \%$ of the total. The explanation for this is unclear but correlated with the high taurine levels in liver and sera of neonates compared to their mothers' (5).

According to the present data, the $G / T$ ratio was less than one in some cases and the average was low $(3.0 \pm 3.1)$ in the neonatal period, but rose rapidly to adult levels $(6.0 \pm 5.6) 1-3$ months after birth. This is in agreement with the report that the G/T ratio of bile acids in the gallbladder bile reaches the adult level 2-7 months afte birth (5). Biliary conjugates of bile acids showed a large excess of taurine/glycine in neonatal life $(5,19)$, but the serum levels of glycine conjugated bile acids predominated over taurine conjugated bile acids. This may be due to selectivity of intestinal absorption or hepatic uptake of bile acids. Furthermore, in this study, the infants were mainly bottle-fed, which might explain why the $\mathrm{G} / \mathrm{T}$ ratio was not still lower.

The $S / P$ ratio showed low levels for about 1 yr after birth and increased thereafter, probably due to increasing intestinal bacteria performing $7 \alpha$-dehydroxylation (8). Although in previous literature, studies of UDCA in children is scanty, it is notable that UDCA was detected in sera of normal children and showed no developmental changes.

HPLC employed in this study has made it possible to measure very small amounts of glycine and taurine conjugated bile acids in serum, determination of which has hitherto been difficult. However, it failed to demonstrate sulfate conjugated bile acids and $3 \beta$-hydroxy-5-cholenoic acid found in serum of normal individuals in small amounts. The $3 \alpha$-sulfate esters prevented the determination of sulfate conjugated bile acids, but it is possible to determine them with a solvolysis procedure. The $3 \beta$ hydroxy-5-cholenoic acid can be determined if we use a column packed with immobilized $3 \beta$-hydroxysteroid dehydrogenase instead of a column packed with immobilized $3 \alpha$-hydroxysteroid dehydrogenase.

Changes noted during development include a change of the 
primary bile acid from GCA to GCDCA, a decrease in the amounts of taurine conjugated bile acids and a tendency toward increasing amounts of unconjugated bile acids and secondary bile acids.

Acknowledgments. I express my thanks to Emeritus Prof. Hideo Kato, Prof. Keijiro Yabuta, Dr. Hiroshi Nittono, Department of Pediatrics Juntendo University School of Medicine, and Associate Prof. Birgitta Strandvik, Department of Pediatrics Karolinska Institutet, for constructive criticism of the manuscript, to Mr. Sakae Higashidate of Japan Spectroscopic Co., Ltd. (Jasco) for his assistance on HPLC, to Miss Noriko Furuta of our research laboratory for her assistance, and to Mr. Archibald M. Brown for correcting the English text.

\section{REFERENCES}

1. Arisaka $O 1981$ Studies on the enterohepatic circulation of bile acids in infancy and childhood. Part 1. Enterohepatic circulation of cholic acid in hepatobiliary disorders of children-especially its application for differential diagnosis of neonatal hepatitis and congenital biliary atresia. Acta Paediatr Jpn. 23:341

2. Arisaka O 1981 Studies on the enterohepatic circulation of bile acids in infancy and childhood. Part 2. The effects of free and conjugated chenodeoxycholic acid on enterohepatic circulation of bile acids-experimental study in rabbits, Acta Paediatr Jpn 23:353

3. Back P, Ross K 1973 Identification of $3 \beta$-hydroxy-5-cholenoic acid in human meconium. Hoppe-Seyler's Z Physiol Chem 354:83

4. Barbara L, Lazzari R, Roda A, Aldine R, Festi D, Sama L, Morselli M, Mcollina A, Bazzoli F, Mazzalia G, Roda E 1980 Serum bile acids in newborns and children. Pediatr Res 14:1222

5. Challacombe DN, Edkins S, and Brown GA 1975 Duodenal bile acids in infancy. Arch Dis Child 50:837

6. Danielsson H, and Rutter, W. J. 1968 The metabolism of bile acids in the developing rat liver. Biochemistry 7:346

7. Encranz JC, Sjövall J 1959 On the bile acids in duodenal contents of infants and children. Clin Chem Acta 4:793

8. Hayakawa S, and Samuelsson B 1964 Transformation of cholic acid in vitro by Corynebacterium simplex. J Biol Chem 239:94

9. Heikura S, Similä, S Finni, K Mäentausta O, and Jänne O. 1980 Cholic acid and chenodeoxycholic acid concentration in serum during infancy and childhood. Acta Paediatr Scand 69:659

10. Hofmann, A. F. 1977 The enterohepatic circulation of bile acids in man. Clin Gastroenterol 6:3

11. Holsti P 1956 Experimental cirrhosis of the liver in rabbits induced by gastric instillation of desiccated whole bile. Acta Pathol Microbiol Scand 113: (suppl): 1

12. Holsti $P 1960$ Cirrhosis of the liver induced in rabbits by gastric instillation of 3-monohydroxycholanic acid. Nature, 186:250

13. Jenner RH, and Howard ER 1975 Unsaturated monohydroxy bile acids as a cause of idiopathic obstructive cholangiopathy. Lancet 2:1073

14. Kato H, and Nittono $H 1980$ Toxic effects of bile acids on the hepatobiliary system. In: Cholestasis in Infancy-Its Pathogenesis, Diagnosis and Treatment. Japan Medical Research Foundation, University of Tokyo Press Tokyo

15. Makino I, Nakagawa S, Mashimo K. 1969 Conjugated and unconjugated serum bile acid levels in patients with hepatobiliary diseases. Gastroenterology 56:1033

16. Makino I, Sjövall J, Norman A, and Strandvik B: Excretion of $3 \beta$-hydroxy-5cholenoic and $3 \alpha$-hydroxy-5 $\alpha$-cholanoic acids in urine of infants with biliary atresia. FEBS Lett 15:161

17. Midvedt T, and Norman A 1967 Bile acid transformation by microbial strains belonging to genera found in intestinal contents. Acta Pathol Microbiol Scand 71:629

18. Nittono H 1979 Studies on the toxicity of bile acids in infants. Part 1. Effect of orally fed bile acids on the hepatobiliary system in rabbits.Acta Paediatr Jpn 20:11

19. Nittono $H$, Niijima S, Ohazama $H$, Sasaki $H$, Arisaka O, and Yamada $K$ Conjugated bile acid levels in gallbladder biles of premature babies, newborn babies and infants.Nippon Shonikagakkai Zasshi 85:224

20. Okuyama S 1979 HPLC analysis of individual bile acids: free, glycine-and taurine-conjugated bile acids. Gastroenterol Jpn 14:129

21. Okuyama S 1981 Analysis of free, glycine-and taurine-conjugated individual bile acids using HPLC and immobilized $3 \alpha$-hydroxysteroid dehydrogenase in column form. Jap J Clin Pathol 29:446

22. Sandberg DH, Sjövall J, Sjövall K, and Turner DA 1965 Measurement of human serum bile acids by gas-liquid chromatography. J Lipid Res 6: 182

23. Sjövall $J 1959$ The occurrence of $7 \beta$-hydroxylated bile acids in human bile. Acta Chem Scand 13:711

24. Strandvik B 1973 Bile acid metabolism in cholestasis of infancy. Opuseul Med Suppl 29:1

\title{
Exclusively Breast-Fed Healthy Infants Grow Slower than Reference Infants
}

\author{
LEENA SALMENPERÄ, JAAKKO PERHEENTUPA, AND MARTTI A. SIIMES \\ Children's Hospital, University of Helsinki, Helsinki, Finland
}

\begin{abstract}
We have studied the nutritional adequacy of exclusive breast-feeding by following prospectively the growth and protein nutrition of healthy infants during the 1st yr of life. The number of exclusively breast-fed infants was 116 at the age of 6 months and 36 at 9 months. These infants had slower length velocity after age 3 months than a comparison group of 32 infants who were weaned early and given formula plus solids. As a group, the exclusively breast-fed infants lagged slightly, but progressively, behind in relative length. By 9 months, $45 \%$ of them versus $18 \%$ of the comparison group showed a $>1$ SD decrease in relative length. No such decrease was found in relative weight. Skinfolds and weight for length ${ }^{2}$ index showed that
\end{abstract}

Received May 21, 1984; accepted November 1, 1984.

Address correspondence to Leena Salmenperä, M.D., Children's Hospital, SF00290 Helsinki

This study was supported by grant from Sigrid Jusélius Foundation and the Foundation for Paediatric Research, Helsinki, Finland and financial support from Valio Co Ltd, Helsinki, Finland. they were heavier for their length than the comparison infants. At 6 and 9 months the calculated protein intake $(0.9 \mathrm{~g} / \mathrm{kg} / \mathrm{day})$ was much less than the recommended amount $(2.0 \mathrm{~g} / \mathrm{kg} /$ day $)$. Serum prealbumin concentration was lower than in the comparison group but this was noted as early as $\mathbf{4}$ months. No relation was found between the parameters of growth and protein nutrition either individually or in general. Whether the slower growth of the exclusively breast-fed infants represents appropriate physiological growth or whether it indicates nutritional deficiency is not known but we did not find any evidence of protein deficiency. Six infants did, however, show subsequent catch-up growth which could indicate previous malnutrition. (Pediatr Res 19: 307-312, 1985)

\section{Abbreviation}

SDS, standard deviation score, relative length/weight 